Document downloaded from:

http://hdl.handle.net/10251/52488

This paper must be cited as:

Fernández González, HA.; Rodrigo Peñarrocha, VM.; Rubio Arjona, L.; Reig, J. (2014). Path loss characterization in vehicular environments under LOS and NLOS conditions at 5.9 GHz. En Antennas and Propagation (EuCAP), 2014 8th European Conference on. Institute of Electrical and Electronics Engineers (IEEE). 3044-3048.

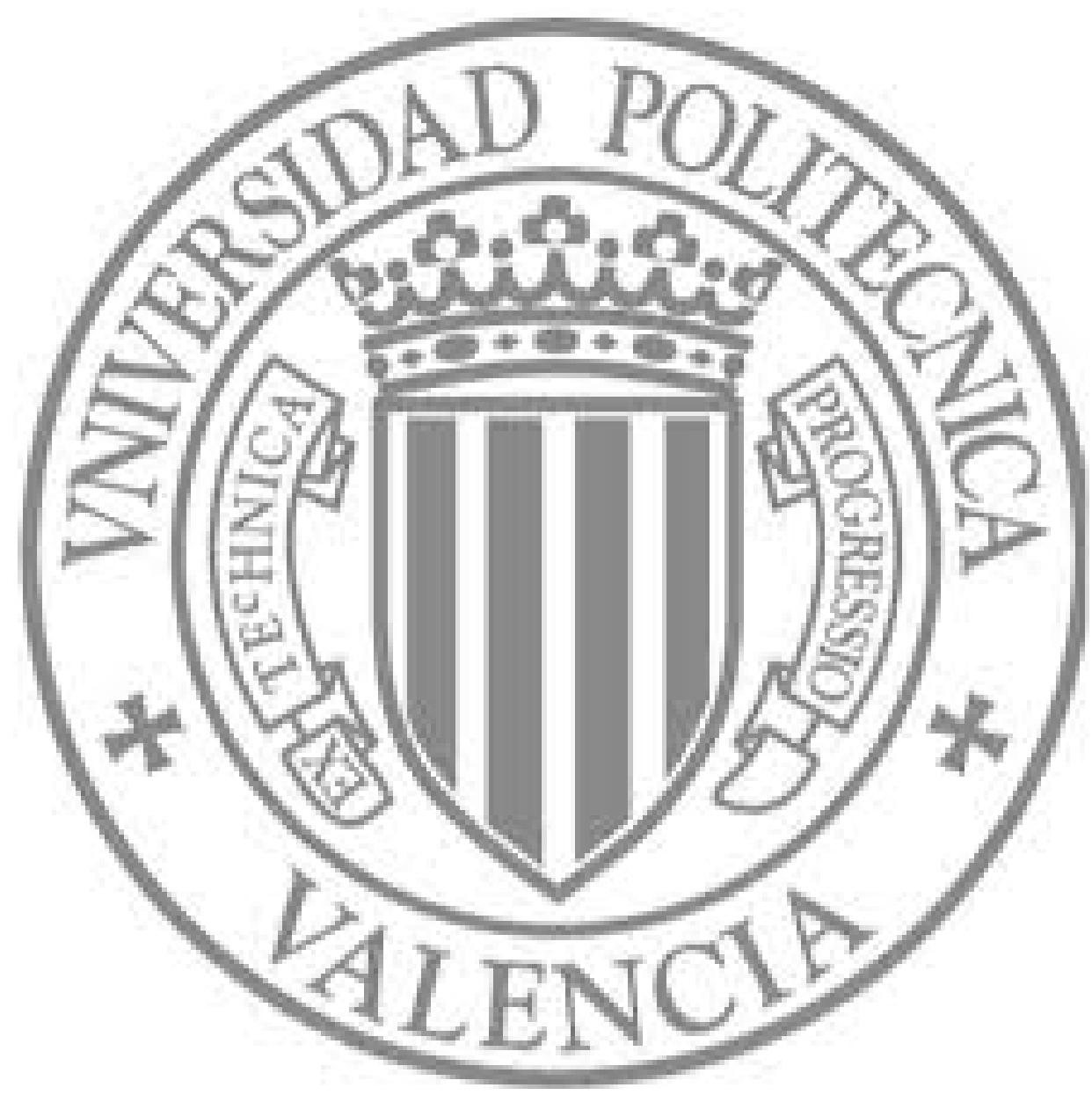

The final publication is available at

http://ieeexplore.ieee.org/xpl/mostRecentlssue.jsp?punumber=6888545

Copyright Institute of Electrical and Electronics Engineers (IEEE) 


\title{
Path loss characterization in vehicular environments under LOS and NLOS conditions at $5.9 \mathrm{GHz}$
}

\author{
Herman Fernández ${ }^{1}$, Vicent M. Rodrigo-Peñarrocha ${ }^{2}$, Lorenzo Rubio ${ }^{2}$, Juan Reig ${ }^{2}$ \\ ${ }^{1}$ Escuela de Ingeniería Electrónica, Universidad Pedagógica y Tecnológica de Somagoso, Colombia \\ ${ }^{2}$ Departamento de Comunicaciones, Universidad Politècnica de València, Valencia, Spain \\ herman.fernandez@uptc.edu.co;\{vrodrigo,lrubio,jreigp\}@dcom.upv.es
}

\begin{abstract}
Based on a narrowband channel measurement campaign, this paper will characterize the path loss in vehicular environments under line-of-sight (LOS) and non-LOS (NLOS) at $5.9 \mathrm{GHz}$. Based on a linear slope model, values of the path loss exponent and the standard deviation of the shadowing will be reported in three different environments, i.e., urban with high road traffic density, urban with moderate/low traffic density, and expressway. The results presented here can be incorporated in an easy way into vehicular networks (VANETs) simulators to develop, evaluate and validate new protocols and systems architectures configurations under more realistic propagation conditions.
\end{abstract}

Index Terms-Vehicular channels, vehicular communications, channel measurements, path loss characterization.

\section{INTRODUCTION}

In recent years, vehicular communications are receiving considerable attention from the automobile industry, governments and academic institutions. New vehicular safety proposals based on the integration of information and communication technologies into both vehicles and infrastructure are being developed. These proposals have led to the intelligent transportation system (ITS) concept $[1,2]$. Some of these safety proposals require large-range vehicular communications systems. In this context, the introduction of vehicular ad hoc networks (VANETs), as a special case of vehicular communications, can extend the communication distance and provide more information to drivers in real-time. Nevertheless, vehicular communications can also provide non-safety applications, such as vehicular high speed Internet access and in-vehicle entertainment applications, among others. Government radio management applications have allocated specific bands for the deployment of ITS applications. For instance, the Federal Communication Commission (FCC) assigned $75 \mathrm{MHz}$ of licensed spectrum at the $5.9 \mathrm{GHz}$ frequency band (from 5.850 $\mathrm{GHz}$ to $5.925 \mathrm{GHz}$ ), as a part of ITS for dedicated short-range communications (DSRC). In Europe, the European Telecommunication Standard Institute (ETSI) adopted the DSRC band for ITS applications assigning $50 \mathrm{MHz}$ (from $5.875 \mathrm{GHz}$ to $5.925 \mathrm{GHz}$ ). The special features of both safety and non-safety applications require to develop and implement new communication technologies, where one of the many challenges to be address is the characterization and modeling of the vehicular propagation channel [2-6]. Thus, the vehicular channel is at present an important area in channel modeling. The mobility of both terminals, the transmitter $(\mathrm{Tx})$ and the receiver $(\mathrm{Rx})$, the use of low elevation antennas and the frequency band operation make that vehicular-to-vehicular $(\mathrm{V} 2 \mathrm{~V})$ systems differ from the traditional fixed-to-mobile (F2M) or cellular systems. Thus channel models developed for F2M systems are not suitable in the deployment of future $\mathrm{V} 2 \mathrm{~V}$ communication systems. In this context, new channel models and path loss characterization are essential to evaluate and validate networking protocols under realistic propagation conditions.

Vehicular propagation channel characteristics are related to the type of environment (i.e., urban, suburban, expressway, etc), vehicles speed and vehicular road traffic density. Due to the mobility of terminals, the line-of-shight (LOS) can suffer blocking effects, alternating to non-LOS conditions. Based on a narrowband channel measurement campaign carried out at $5.9 \mathrm{GHz}$, this paper will characterize the path loss in three different vehicular environments under LOS and NLOS conditions and in real driving behaviors.

The final paper will be organized as follows: Section II will describe the measurement setup and the measured scenarios. Section III will present the measurement results and path loss characterization. Finally, the conclusions will be given in Section IV.

\section{Channel MEASUREMEnts}

\section{A. Measurement setup}

To carry out the path loss characterization of the vehicular propagation channel, we have performed narrowband channel measurements. We have used a signal generator (SG) at the Tx side (Tx vehicle) transmitting a continuous wave $(\mathrm{CW})$ at 5.9 GHz. A high power amplifier (HPA) was used to achieve an equivalent isotropically radiated power (EIRP) equal to +23 $\mathrm{dBm}$. At the $\mathrm{Rx}$ side ( $\mathrm{Rx}$ vehicle) we have used a vector network analyzer (VNA) to measure the received signal level through the $b_{2}$ parameter. The $b_{2}$ parameter was continuously measured using traces of $N=5000$ test points, with an average acquisition time per test point of $45 \mu$ s for an intermediated filter (IF) equal to $100 \mathrm{kHz}$. With this configuration, the integration period of the measured signal level is equivalent to a vehicle displacement of $60 \lambda$ at $13.88 \mathrm{~m} / \mathrm{s}(50 \mathrm{~km} / \mathrm{h})$. Thus the average of the measured samples in each trace permits us to remove (filteting) the shor-term fading fluctuations. As a result, the fluctuations of the measured power are due to blocking 


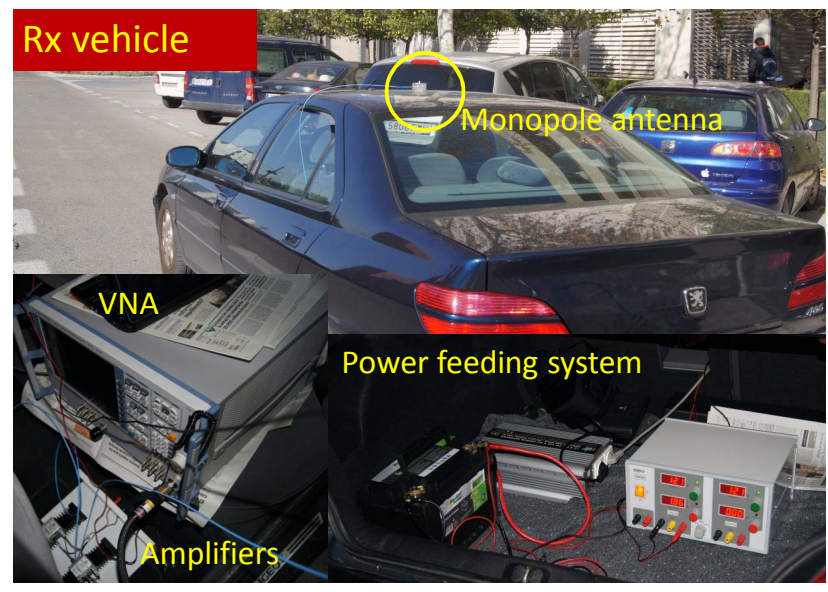

Fig. 1. View of the equipment installed in the $\mathrm{Rx}$ vehicle.

(shadowing) effects of the interacting vehicles between the Tx and Rx. Two medium high power amplifiers (MPAs) at the Rx and low-loss cables were also used to achieve a total gain system of about $86.67 \mathrm{~dB}$. A laptop was used to automate the measurements acquisition system and record the measured data. Both Tx and Rx used the same antenna, a $\lambda / 4$ monopole roof-mounted in the center of the vehicles through a magnetic base and transmitting with vertical polarization. In addition to the radio frequency (RF) equipment, both Tx and $\mathrm{Rx}$ were equipped with GPS receivers, each one controlled by a laptop, to provide information about the acquisition time of measurements, as well as relative speed and separation distance between the Tx and Rx vehicles. Fig. 1 shows a view of the equipment installed in the Rx vehicle.

\section{B. Measurement scenarios}

The type of environment, the vehicles speed and the vehicular road traffic density condition the propagation characteristics of the vehicular channel. Thus road traffic densities are usually higher in urban environments and higher vehicles speed are given in expressway and highway environments. In order to characterize the path loss taking into account these features, we have performed measurements in three different scenarios: (i) urban with high road traffic density, (ii) urban with moderate/low road traffic density,and (iii) an expressway environment with high road traffic density. A morphological description of the measured scenarios will be presented in the final version of the paper.

\section{Measurement Results And PATH loss CHARACTERIZATION}

\section{A. Methodology}

In vehicular communications the shadowing is mainly due to the intermittent blocking of the LOS condition by vehicles between the Tx and Rx. To distinguish between LOS and NLOS, we incorporated a video camera to the $\mathrm{Rx}$ vehicle (see Fig. 2). In the measurements the Tx vehicle circulated back. Thus during the processing of the measures we can classify

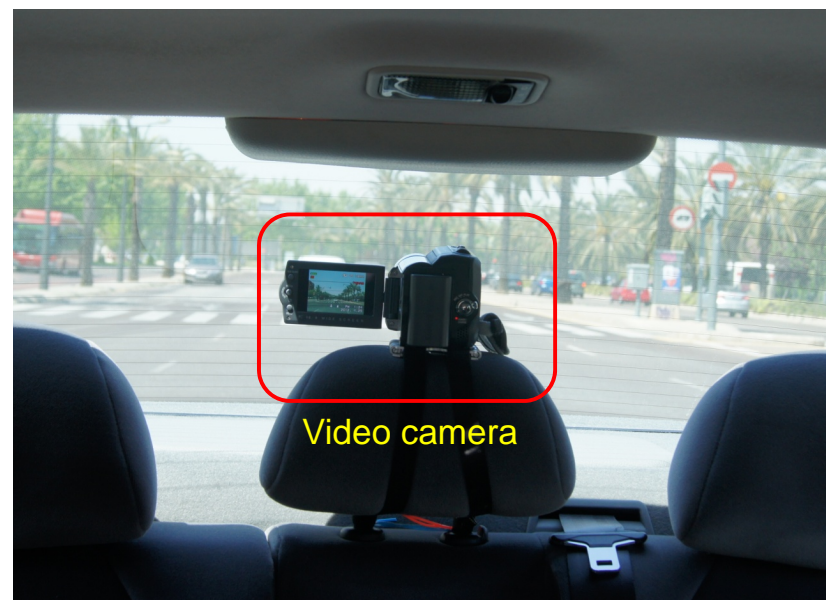

Fig. 2. Video camera on board the Rx vehicle.

the subpaths with LOS and NLOS propagation conditions. To perform this classification was necessary to synchronize the video camera to the laptops through its timebase. For each subpath measured, a linear relationship between the path loss, expressed in $\mathrm{dB}$, and the logarithmic of the Tx-Rx separation distance can be established, in the same manner that the traditional fix-to-mobile (F2M) channels. Then the path loss, denoted by $P L$, is estimated by the following linear model $[2,6]$ :

$$
P L(d)=P L_{0}+10 \gamma \log _{10}(d)+S, \quad d \geqslant d_{\text {min }},
$$

where $d$ is the Tx-Rx separation distance, $P L_{0}$ is the path loss at the reference distance $d_{m i n}, \gamma$ is the path loss exponent related to the propagation scenario characteristics, and $S$ is a gaussian random variable with zero mean and standard deviation $\sigma_{S}$, used to model the large scale fading. From (1), this model is restricted to $\mathrm{Tx}-\mathrm{Rx}$ separation distances exceeding $d_{\text {min }}$. In our measurements, $d_{\min }$ ranges from 5 to $8 \mathrm{~m}$.

\section{B. Previous results}

Fig. 3 shows the evolution of Tx-Rx separation distance, the vehicles speed and the measured power level over time in a record of $180 \mathrm{~s}$. In the figure, we have circled two subpaths that correspond to LOS and NLOS conditions, showing that fading depths are generally higher in NLOS.

Table I summarized the values of the path loss model parameters derived from the urban scenario with high road traffic density. These values were obtained using the information reported by the video camera.

In LOS conditions, the path loss exponent ranges from 1.29 to 2.17 with a mean value of 1.69 . For NLOS conditions the values of the path loss exponent are higher, ranging from 1.14 to 3.02 , with a mean value equal to 1.90 . With respect to the $P L_{0}$ parameter, it exhibits a greater fluctuation in NLOS conditions. We have observed from the measured data that a path loss exponent lower than 2 not necessarily corresponds 

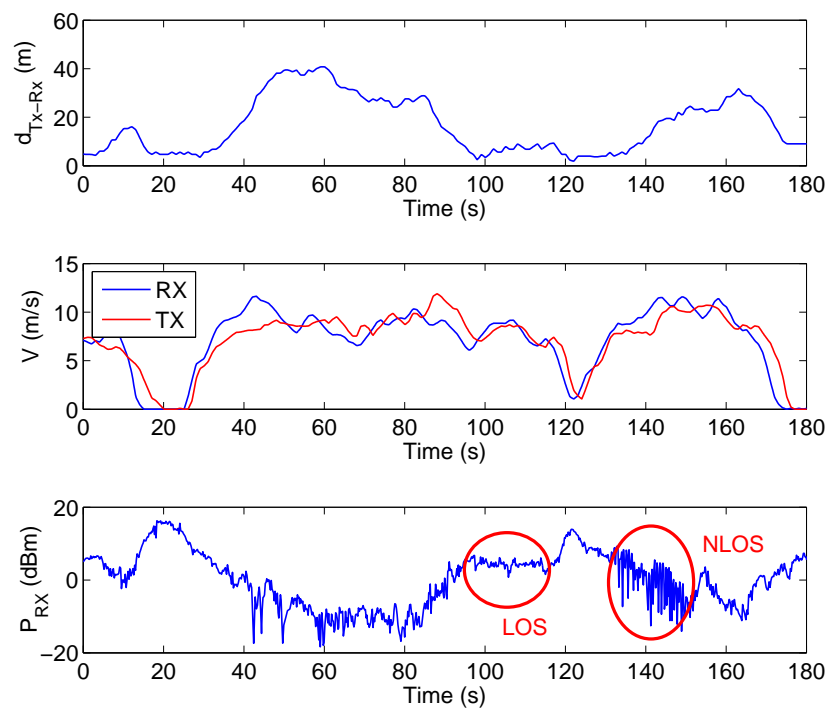

Fig. 3. (a) Tx-Rx separation distance, (b) vehicles speed, and (c) measured power level over time.

TABLE I

PATH LOSS MODEL PARAMETERS USING THE VIDEO CAMERA.

\begin{tabular}{ccccc}
\hline & Value & $\gamma$ & $L_{0}(\mathrm{~dB})$ & $\sigma_{S}(\mathrm{~dB})$ \\
\hline \multirow{4}{*}{ LOS } & Maximum & 2.17 & 55.89 & 1.98 \\
& Mean & 1.69 & 51.22 & 1.82 \\
& Minimum & 1.29 & 44.41 & 1.61 \\
\hline \multirow{2}{*}{ NLOS } & Maximum & 3.02 & 66.77 & 2.79 \\
& Mean & 1.90 & 52.31 & 2.35 \\
& Minimum & 1.14 & 37.33 & 1.90 \\
\hline
\end{tabular}

to propagation conditions better than free space. Generally, values of the path loss exponent lower than 2 are related to $P L_{0}$ values greater than free space.

In the final version of the paper we will analyze in more detail the path loss and will present the results of all measured scenarios. The results will be compared to those in which no distinction is made between LOS and NLOS, as well as to other works published in the literature.

\section{CONCLUSION}

Based on a narrowband channel measurement campaign carried out at $5.9 \mathrm{GHz}$ (the DSRC frequency band), the paper will analyze the path loss in three different environments (i.e., urban with high road traffic density, urban with moderate/low road traffic density, and expressway). For a linear path loss model, the values of the model parameters will be extracted from the measured data, distinguishing between LOS and NLOS conditions. These values can be easily incorporated into VANETs simulator in order to take into account more realistic propagation conditions, and can also be used in the planning and deployment of future vehicular networks.

\section{ACKNOWLEDGMENT}

The authors want to thank J. A. Campuzano, D. Balaguer and L. Moragón for their support during the measurement campaigns, as well as B. Bernardo-Clemente and A. VilaJiménez for their support and assistance in the laboratory activities.

\section{REFERENCES}

[1] B. Gallager, H. Akatsuka and H. Suzuki, "Wireless communications for vehicle safety: radio link performance and wireless connetivity," IEEE Vehicular Technology Magazine, vol.1, no. 4, pp. 4-24, 2006.

[2] L. Rubio, J. Reig and H. Fernández, Propagation aspects in vehicular networks, Vehicular technologies. Miguel Almeida (Ed), InTech, 2011.

[3] D. G. Michelson and S. S. Ghassemzadeh, New directions in wireless communications, Springer Science+Busines Media, 2009.

[4] J. Karedal, N. Czink, A. Paier, F. Tufvesson and A. F. Molisch, "Path loss modeling for vehicle-to-vehicle communications," IEEE Transactions on Vehicuar Technology, vol. 60, no.1, pp. 323-327, 2011.

[5] L. Cheng, B. Henty, D. Stancil, F. Bai and P. Mudalige, "Mobile vehicleto-vehicle narrow-band channel measurement and characterization of the $5.9 \mathrm{GHz}$ dedicated short range communication (DSRC) frequency band." IEEE Journal on Selected Areas in Communications, vol. 25, no. 8, pp. 1501-1516, 2007.

[6] H. Fernández, L. Rubio, J. Reig, V. M. Rodrigo-Peñarrocha and A. Valero, "Path loss modeling for vehicular system performance and communication protocols evaluation," International Journal of Mobile Networks and Applications, In press, 2013. 